\title{
EVALUACIÓN DE LA CALIDAD DEL AGUA FLUVIAL CON DIATOMEAS (Bacillariophyceae), UNA EXPERIENCIA EN TACNA, PERÚ
}

\author{
José Calizaya-Anco1,a, Miriam Avendaño-Cáceres²,b, Irma Delgado-Vargas²,c
}

\begin{abstract}
RESUMEN
Con el objetivo de evaluar la calidad del agua de la cuenca del río Locumba, Tacna (Perú), se tomaron muestras de agua de diez estaciones ubicadas a lo largo de la cuenca del río Locumba, durante seis periodos en un ciclo anual. Asimismo, se evaluó la diversidad y número de diatomeas y once parámetros fisicoquímicos para determinar el grado de contaminación del agua. Encontramos que conforme se desciende en la cuenca, la diversidad de diatomeas disminuyó de 2,37 bits cel-1 a 0,71 bits cel-1 y el gradiente de contaminantes se incrementó. Además, con este incremento, se observó un aumento en el número de especies tolerantes a altos niveles de perturbación ambiental. Se observó un incremento en todos los parámetros fisicoquímicos empleados para evaluar el grado de contaminación. Se sugiere que las diatomeas pueden ser adecuados bioindicadores al momento de evaluar la calidad de agua en esta cuenca.
\end{abstract}

Palabras clave: Limnología; Caudal de agua del río; Diatomeas; Contaminación de ríos; Ecosistema (fuente: DeCS BIREME).

\section{EVALUATION OF THE QUALITY OF RIVER WATER WITH DIATOMS (Bacillariophyceae): AN EXPERIENCE IN TACNA, PERU}

\begin{abstract}
In order to evaluate the quality of the water of the Locumba river, Tacna (Peru), water samples were taken from ten stations located along the Locumba river basin, during six periods in an annual cycle. The diversity and number of diatoms was also evaluated, together with eleven physiochemical parameters in order to determine the degree of water contamination. We found that as the basin advanced down the mountain, the diversity of diatoms decreased from 2.37 bits cell- 1 to 0.71 bits cell-1 and the gradient of contaminants increased. In addition to this increase, the number of species tolerant to high levels of environmental disturbance rose. An increase in all physiochemical parameters used to evaluate the degree of contamination was observed. These results suggest that diatoms can be adequate bioindicators when evaluating the quality of water in this basin.
\end{abstract}

Key words: Limnology; Stream flow; Diatoms; River pollution; Ecosystem (source: MeSH NLM).

\section{INTRODUCCIÓN}

En el Perú, como en otros países sudamericanos, los ríos recorren extensas zonas altoandinas hasta llegar a la costa arrastrando sedimentos característicos de la cuenca, además de aquellos procedentes de efluentes de aguas residuales de alcantarillado, industrias y disoluciones de agroquímicos provenientes de zonas agrícolas. Todos estos procesos condicionan un sistema altamente heterogéneo, con un marcado gradiente de polutantes que se manifiestan en una variación de la química del agua en intervalos cortos de tiempo y de escalas espaciales (1), que requiere del desarrollo de metodologías adecuadas para su evaluación. En la actualidad se realizan análisis fisicoquímicos que emplean equipos sofisticados, los cuales pueden detectar incluso

\footnotetext{
Universidad Privada de Tacna. Tacna, Perú

Laboratorio de Salud Pública. Tacna, Perú

a Biólogo microbiólogo; ${ }^{\mathrm{b}}$ ingeniera en Industrias Alimentarias; ${ }^{\mathrm{C}}$ técnico en laboratorio clínico

* Este artículo se basa en el informe técnico "Diatomeas de la cuenca del río Locumba como posibles bioinidicadores de la calidad del agua" publicado en la página web del INS (Serie de Informes Técnicos $N^{\circ}{ }^{\circ} 5,2007$ )

Recibido: 06-08-12 Aprobado: 17-10-12
}

Citar como: Calizaya-Anco J, Avendaño-Cáceres M, Delgado-Vargas I. Evaluación de la calidad del agua fluvial con diatomeas (Bacillariophyceae), una experiencia en Tacna, Perú. Rev Peru Med Exp Salud Publica. 2013;30(1):58-63. 
trazas de componentes orgánicos e inorgánicos, pero cuyos resultados solo reflejan la condición del momento de la toma de muestra.

Ante estas limitaciones, varios países han desarrollado alternativas, como la Directiva Marco del Agua de la Unión Europea, que recomienda el uso de organismos como los peces, macroinvertebrados y microalgas como herramientas en la evaluación del estado ecológico de aguas superficiales ${ }^{(2)}$. Las microalgas podrían servir como indicadores de cambios ambientales de muchos ecosistemas acuáticos debido a su elevada sensibilidad a un amplio rango de contaminantes. Sus ciclos de vida son cortos y las hacen indicadoras adecuadas para evaluar impactos a corto plazo y, además, la colecta es relativamente sencilla.

Existen dos importantes conceptos a favor de una evaluación biológica; primero, los organismos tienen una respuesta integradora con respecto a su medioambiente y a las fluctuaciones en la calidad del agua, los cuales no son detectados por análisis químicos intermitentes; y segundo, supervisar la evolución de estas especies permite evaluar si el ecosistema acuático mantiene condiciones saludables que conserven la diversidad de las comunidades de organismos ${ }^{(3)}$.

Las diatomeas son las microalgas más numerosas del fitobentos y fitoplancton que debido a sus características biológicas y ecológicas reflejan adecuadamente el estado ecológico de las aguas superficiales frente a impactos antrópicos ${ }^{(4)}$. Así también proporcionan información valiosa de la condición del ecosistema acuático al igual que los indicadores animales (macroinvertebrados y peces); permiten realizar una adecuada y confiable línea base como referencia, y su estudio es más costo-efectivo ${ }^{(5)}$.

En Perú, las investigaciones ficológicas relacionadas con bioindicadores presentan un distanciamiento entre el estudio de las microalgas y sus parámetros ambientales, debido a que en estos últimos las microalgas son tomadas como referencia en la caracterización del agua y no son consideradas como influyentes en su distribución poblacional. Peor aun, no se considera a las microalgas como herramienta en la caracterización de un ambiente acuático ${ }^{(6)}$. Tampoco hay una exigencia legal para su utilización, debido a que la Ley de Recursos Hídricos (Ley 29338), y los Estándares Nacionales de Calidad Ambiental para agua (D. S. 002-2008-MINAM) contemplan los análisis físicos, químicos y microbiológicos (bacterias y protozoarios parásitos) como los indicadores de la calidad del agua en ecosistemas acuáticos naturales.

La cuenca del río Locumba, ubicada entre los $70^{\circ} 06^{\prime}$ y $71^{\circ}$ $05^{\prime}$ de longitud oeste y $16^{\circ} 47^{\prime}$ y $17^{\circ} 54^{\prime}$ de latitud sur, tiene una extensión de 5742 km², de las cuales 505 km² corresponden a la cuenca localizada por encima de los 3900 $\mathrm{m}$ de altitud (cuenca húmeda) y que aporta los recursos hídricos al escurrimiento superficial que tiene sus nacientes en la parte alta de la región. Pertenecen a esta cuenca las formaciones geológicas Capillune, Toquepala y Moquegua, de rocas sedimentarias volcánicas de erosión glaciar, rocas volcánicas con intercalaciones escasas de sedimentos clásticos y de rocas sedimentarias continentales respectivamente.

A lo largo de la cuenca del río Locumba, potencialmente puede haber un incremento en la gradiente de polutantes producto de factores antrópicos, por lo cual se hace necesario la evaluación de los indicadores de cambios ambientales. Por todo ello, el objetivo de esta investigación es evaluar la calidad del agua en la cuenca del río Locumba y asociar el estado de contaminación de la cuenca con los cambios en la cantidad y riqueza específica de especies de diatomeas.

\section{EL ESTUDIO}

Se establecieron diez estaciones a lo largo de la cuenca del río Locumba (Figura1), tomando como criterio de selección la gradiente de contaminantes vertidos a lo largo de la cuenca por la actividad humana, estas fueron: manantial Chaullapujo $(\mathrm{CH})$; arroyo Tacalaya (TA); río Callazas (CA); río Salado (SA); filtración Curibaya $(\mathrm{CU})$; río llabaya (YL); puente Locumba (PL); desagüe Locumba (DL); puente Panamericana (PP), y desagüe Leche Gloria (LG) [anexos disponibles en www.rpmesp. ins.gob.pe]. En ellas se realizó seis campañas de muestreo en un ciclo anual, haciendo un total de 59 muestras (la muestra no tomada se debió a que una estación no tenía agua en la fecha en que correspondió la toma).

Los análisis fisicoquímicos y microbiológicos se realizaron bajo métodos estandarizados según las normas de la American Public Health Association (APHA) y de la American Water Works Association (AWWA) de 1999; los parámetros evaluados fueron: coliformes totales, sólidos totales en suspensión, demanda bioquímica de oxígeno $\left(\mathrm{DBO}_{5}\right)$, demanda química de oxígeno (DQO), arsénico, salinidad, temperatura, turbidez, conductividad, dureza total y $\mathrm{pH}$. Según la APHA, para la evaluación de los coliformes totales se empleó el método "número más probable" (nmp), dado que para esta medición se empleó un medio líquido.

Las muestras de diatomeas fueron obtenidas a partir del raspado superficial de rocas presentes en el lecho del río (perifiton epilítico); se raspó por triplicado áreas de 5 a $10 \mathrm{~cm}^{2}$ por roca con un cepillo dental. Las mues- 


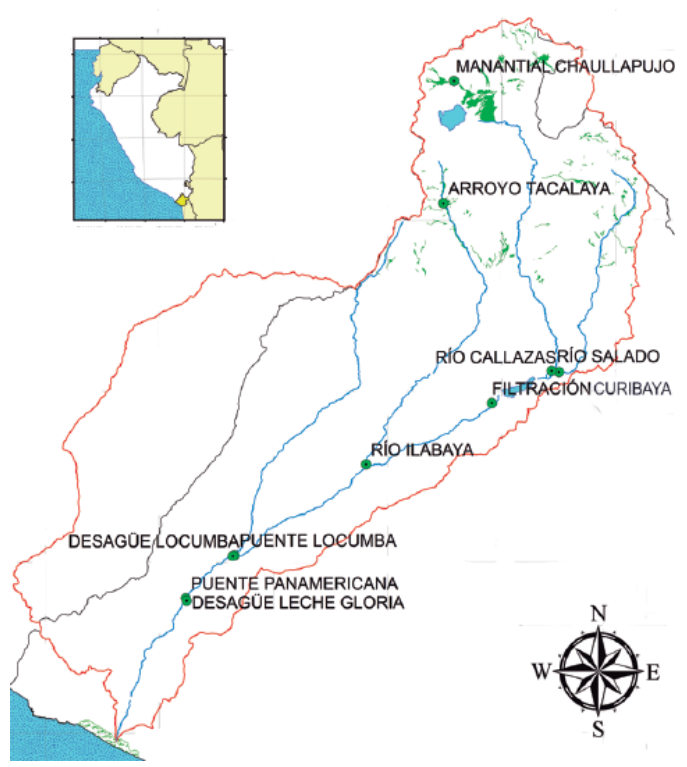

Figura 1. Mapa del área de estudio, se muestran las estaciones de muestreo.

tras fueron conservadas para su transporte en formol al $4 \%$ en frascos herméticos de $100 \mathrm{~mL}$, con $\mathrm{pH}$ neutro, debidamente etiquetados con fecha y lugar de muestreo ${ }^{(7)}$. Para la identificación de las diatomeas se oxidó la muestra en un vaso de precipitados de $500 \mathrm{~mL}$ con una doble proporción de peróxido de hidrógeno concentrado, y se dejó hervir hasta que la mezcla adopte un color gris. Posteriormente, con una alícuota de esta mezcla se preparó una lámina permanente con la resina sintética Zrax ${ }^{\circledR}$ (índice de refracción mayor a 1,7) ${ }^{(8)}$. Cada lámina fue observada a un aumento de $1600 \mathrm{x}$ en un microscopio Leica DME de 1,25 de apertura numérica. Para la identificación se utilizaron claves taxonómicas especializadas ${ }^{(9-13)}$. Se realizó un conteo promedio de 400 valvas por cada muestra.
Para evaluar el nivel de perturbación de las zonas de muestreo propusimos el uso de índices de diversidad, los cuales consideran tanto el número de especies como el número de individuos de cada especie en un determinado lugar. Para este estudio se empleó el índice de diversidad de Shannon-Wienner, el cual considera que todas las especies en una muestra aleatoria de una población infinita están representadas en dicha muestra, este índice se expresa en "bits cel-1" unidad que denota la cantidad de individuos (para nuestro caso diatomeas) que se encuentran en cada muestra.

\section{HALLAZGOS}

En la cuenca, los valores de los parámetros fisicoquímicos fueron incrementándose a medida que se descendía altitudinalmente. Los valores de coliformes totales se incrementaron en 29 011\% desde YL hasta LG; se pudo apreciar, además, que la salinidad aumentó progresivamente desde SA hasta LG alcanzando un incremento total de $349 \%$; la temperatura se incrementó en un $200 \%$ entre TA y PP; la concentración de arsénico en agua se encontró la máxima concentración en TA con $0,6 \mathrm{mg} / \mathrm{L}$; la media para las estaciones comprendidas entre SA y $L G$ fue de $0,26 \mathrm{mg} / \mathrm{dL}$. En la Tabla 1 se muestran los valores promedio de cada estación de muestreo para los demás indicadores evaluados.

Especies de diatomeas. Se identificaron 77 taxas en 59 muestras colectadas, los géneros más diversos fueron Nitzschia, con 19 especies encontradas y Navicula, con siete; se observó cambios en el ensamblaje de las comunidades de diatomeas y sus abundancias relativas, según la gradiente altitudinal y de las condiciones ambientales que presentó cada estación de muestreo, (ver anexos). El índice de diversidad de ShannonWienner mostró que la diversidad promedio es alta

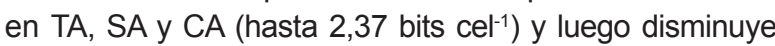

Tabla 1. Valores de los parámetros ambientales en las estaciones de muestreo.

\begin{tabular}{|c|c|c|c|c|c|c|c|c|}
\hline & $\mathrm{pH}$ & $\begin{array}{c}\text { STS } \\
(\mathrm{mgCaCO} / \mathrm{l})\end{array}$ & $\begin{array}{c}\text { Colif_tot } \\
\text { (nmp/100ml) }\end{array}$ & $\begin{array}{l}\text { Turb } \\
\text { (unt) }\end{array}$ & $\begin{array}{c}\text { Dureza } \\
\text { (mgCaCO3/l) }\end{array}$ & $\begin{array}{l}\text { Conduct } \\
(\mathrm{mS} / \mathrm{cm})\end{array}$ & $\begin{array}{c}\text { DQO } \\
\text { (mgO2/l) }\end{array}$ & $\begin{array}{c}\text { DBO5 } \\
\text { (mgO2/l) }\end{array}$ \\
\hline $\begin{array}{l}\text { Manantial } \\
\text { Chaullapujo }\end{array}$ & $8,0 \pm 0,6$ & $0,4 \pm 0,5$ & $3,7 \pm 4,6$ & $0,3 \pm 0,4$ & $39,4 \pm 5,8$ & $2,3 \pm 3,3$ & $14,7 \pm 7,4$ & $5,3 \pm 3,9$ \\
\hline Arroyo Tacalaya & $8,4 \pm 0,5$ & $10,7 \pm 11,6$ & $41,9 \pm 63,8$ & $7,7 \pm 8,5$ & $145,7 \pm 47,8$ & $2,4 \pm 3,2$ & $17,5 \pm 5,8$ & $5,8 \pm 3,7$ \\
\hline Río Callazas & $8,2 \pm 0,4$ & $10,3 \pm 8,2$ & $28,2 \pm 13,8$ & $9,2 \pm 6,1$ & $366,9 \pm 67,0$ & $4,5 \pm 3,2$ & $29,5 \pm 12,0$ & $9,3 \pm 6,0$ \\
\hline Río Salado & $8,5 \pm 0,4$ & $10,4 \pm 7,1$ & $70,5 \pm 78,8$ & $4,5 \pm 4,5$ & $306,5 \pm 27,7$ & $2,4 \pm 2,9$ & $18,7 \pm 11,0$ & $12,3 \pm 8,5$ \\
\hline Filtración Curibaya & $8,4 \pm 0,4$ & $6,4 \pm 14,0$ & $103,2 \pm 75,9$ & $0,5 \pm 0,3$ & $391,8 \pm 35,9$ & $3,3 \pm 2,7$ & $21,0 \pm 11,1$ & $9,8 \pm 5,2$ \\
\hline Río llabaya & $8,5 \pm 0,4$ & $19,0 \pm 29,1$ & $318,6 \pm 353,7$ & $33,0 \pm 69,0$ & $515,8 \pm 57,8$ & $3,4 \pm 2,5$ & $23,8 \pm 23,8$ & $9,6 \pm 4,3$ \\
\hline Puente Locumba & $8,4 \pm 0,4$ & $18,9 \pm 29,7$ & $0,2 \times 10^{4} \pm 0,5 \times 10^{4}$ & $18,0 \pm 27,9$ & $523,3 \pm 28,7$ & $3,7 \pm 2,2$ & $31,8 \pm 27,2$ & $16,5 \pm 12,7$ \\
\hline Desagüe Locumba & $8,4 \pm 0,4$ & $24,3 \pm 36,0$ & $1,1 \times 10^{4} \pm 1,5 \times 10^{4}$ & $17,9 \pm 23,5$ & $524,9 \pm 29,5$ & $3,5 \pm 2,5$ & $34,3 \pm 35,2$ & $19,5 \pm 16,3$ \\
\hline $\begin{array}{l}\text { Puente } \\
\text { Panamericana }\end{array}$ & $8,3 \pm 0,3$ & $2,8 \pm 1,2$ & $348,0 \pm 115,6$ & $1,5 \pm 0,7$ & $570,0 \pm 34,0$ & $4,1 \pm 2,8$ & $35,8 \pm 30,0$ & $15,5 \pm 7,7$ \\
\hline $\begin{array}{l}\text { Desagüe Leche } \\
\text { Gloria }\end{array}$ & $8,0 \pm 0,3$ & $3,9 \pm 4,2$ & $9,2 \times 10^{4} \pm 2,2 \times 10^{4}$ & $1,9 \pm 2,6$ & $805,1 \pm 17,5$ & $5,0 \pm 2,9$ & $34,7 \pm 14,8$ & $18,5 \pm 3,7$ \\
\hline
\end{tabular}

*STS: sólidos totales en suspensión. Colif_tot: coliformes totales. Turb: turbidez. Conduct: conductividad. DQO: demanda química de oxígeno. DBO ${ }_{5}$ : demanda bioquímica de oxígeno durante $\overline{5}$ días. mgO2/l: miligramos de oxígeno por litro. nmp: número más probable. 


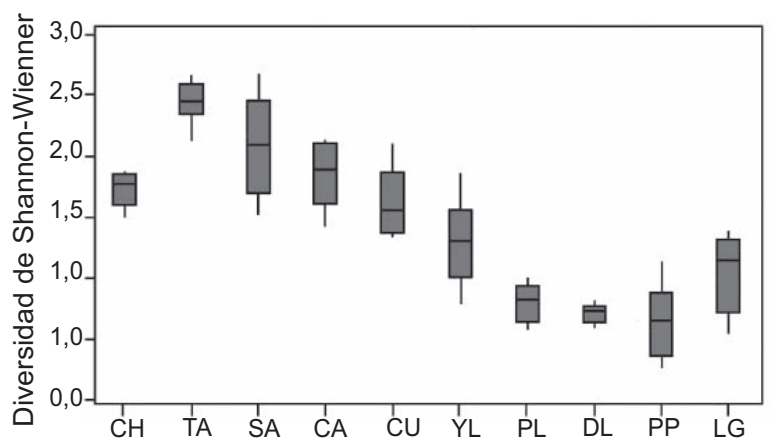

* $\mathrm{CH}$ : manantial Chaullapujo, TA: arroyo Tacalaya, CA: río Callazas, SA: Río Salado, CU: Curibaya, YL: río llabaya, PL: puente Locumba, DL: desagüe Locumba, PP: puente Panamericana y LG: desagüe Leche Gloria. filtración

Figura 2. Índice de diversidad de Shannon - Wienner de acuerdo a estación.

desde la estación CU hasta la estación LG $\left(0,71\right.$ bits cel$\left.^{-1}\right)$, el comportamiento del índice de Shannon-Wienner se muestra en la Figura 2.

Se encontraron cambios en el ensamblaje de las comunidades de diatomeas en el trayecto descendente de la cuenca, (ver anexos). Desde la parte alta de la cuenca ( $\mathrm{CH}$ y $\mathrm{TA})$ donde predomina Diatoma mesodon, Achnanthes minutissima, Achnanthes subatomoides, Fragilaria capucina var. vaucheriae, Cocconeis placentula, Navicula ingapirca, Nitzschia pumila, Planothidium lanceolatum, Navicula lanceolata, Navicula saprophila y Ulnaria ulna consideradas especies de ambientes poco contaminados (oligosaprobios hasta beta-mesosaprobio) ${ }^{(14)}$ hasta YL - LG donde solo predominan las especies Amphora coffeaeformis, Nitzschia frustulum, Gomphonema parvulum, Rhopalodia gibba, Pleurosira laevis, Nitzschia amphibia, Navicula tripunctata, Nitzschia microcephala, Navicula veneta, Nitzschia palea, Navicula atomus, Rhopalodia spp1 y Nitzschia sp cf bergii, conocidas como tolerantes a ambientes fuertemente alterados (alfa-mesosaprobio a polisaprobio) (15). Por otro lado, Reimeria sinuata, Nitzschia pumila, Fragilaria capucina var. vaucheriae, Frankophila similioides, Fragilaria pinnata, Ulnaria ulna, Rhoiscosphenia abbreviata, Fragilaria elliptica, Surirella angusta, Surirella minuta, Nitzschia pusilla y Nitzschia chungara, presentes en TA y Nitzschia capitellata, Navicula lanceolata, Navicula saprophila, Fragilaria brevistriata, Nitzschia ingapirca y Navicula minima, presentes en SA y CA se desarrollaron con baja turbidez $(7,7 ; 9,2$ y 4,5 unt, respectivamente) y son consideradas, en su mayoría, tolerantes a ambientes beta-mesosaprobios. Se encontró a $N$. inconspicua, $N$. palea, $N$. amphibia, $N$. frustulum y $N$. veneta como especies dominantes en estas partes bajas y contaminadas de la cuenca.

\section{DISCUSIÓN}

La concentración de los parámetros ambientales se incrementa conforme al descenso altitudinal de la cuenca, esto se debe a la actividad humana y a las características geológicas de la zona. Los sólidos totales, la turbidez y la conductividad aumentan progresivamente debido al material suspendido proveniente del mismo terreno, y al material orgánico producto de la contaminación de la actividad agrícola, pero principalmente por descarga de aguas contaminadas sin tratamiento ${ }^{(16)}$, esto último confirmado por las altas concentraciones de coliformes totales.

El pH alcalino y el incremento en la dureza del agua se deben a las calizas que promueven la mayor formación de carbonatos y bicarbonatos. Desde el SA hasta el LG los valores de arsénico superan dos veces los estándares de calidad ambiental en agua para la categoría N. ${ }^{\circ} 04$ de la normatividad nacional ${ }^{(17)}$, lo cual sería una condición natural de acuerdo con las características geológicas de la zona, al igual que en el resto de América Latina. La salinidad presente desde SA hasta LG es de origen natural debido a la erosión de rocas sedimentarias que puede verse incrementado en épocas de estiaje o por disminución de su caudal debido a derivaciones del curso natural ${ }^{(18)}$. SA presentó los valores altos de salinidad (342,39 mg/L) y en cuyo lugar desarrollaron especies como Entomoneis paludosa, Tabularia tabulata y Nitzschia hungarica, características de ambientes salobres y poco perturbados con materia orgánica. La $\mathrm{DBO}_{5}$ y la DQO son los dos métodos para medir la cantidad de materia orgánica en el agua ${ }^{(19)}$, por lo que valores altos de la relación $\mathrm{DBO}_{5} / \mathrm{DQO}$ (mayores de 0,4) indicarían materia biodegradable $(\mathrm{CH}$ hasta $\mathrm{CA})$; valores menores (CU hasta $L G$ ) indicarían materia muy difícilmente degradable (como pesticidas orgánicos) que inhiben el desarrollo de diatomeas sensibles a estas condiciones pero adecuadas para Nitzschia inconspicua, Pleurosira laevis y Cocconeis placentula. En nuestro estudio $\mathrm{CH}$ y TA presentaron bajas concentraciones en los parámetros ambientales evaluados, lo cual sugiere una estación con poca perturbación antrópica, esto debido a su ubicación en cabecera de cuenca. Desde YL hasta GL existe concentraciones elevadas de los parámetros ambientales evaluados, lo que explica una condición ambiental muy impactada por la actividad agrícola y por los vertimientos de zonas urbanas.

Tras el análisis del índice de diversidad de ShannonWienner ${ }^{20)}$, que es considerado un buen indicador del impacto que ejerce el ambiente sobre las diatomeas, se sugiere una escala de valores de diversidad para cada nivel de contaminación, que van de 0 a 4 bits cél- ${ }^{1}$, en 
donde de 0 - 1 bits cél-1 existe una contaminación severa, entre $1-2$ bits cél- ${ }^{-1}$ moderada, entre $2-3$ bits cél- ${ }^{-1}$ es leve y entre $3-4$ bits cél- ${ }^{-1}$ la contaminación es imperceptible. Teniendo en cuenta esta escala, los valores bajos de diversidad (0, 24 a 1 bits cel $\left.^{-1}\right)$ obtenidos desde PL hasta $\mathrm{PP}$ indicaron contaminación severa. En tanto que desde SA hasta $\mathrm{YL}$ se encontró contaminación de moderada a leve $\left(1,09\right.$ a 2,12 bits $\left.^{-1}\right)$, y en TA hallamos un ecosistema levemente alterado debido a la carga de nutrientes ${ }^{(21)}$ que se realiza de manera natural. Esto no es aplicable en $\mathrm{CH}$ y en $\mathrm{LG}$, debido a que el primero proviene de zonas hiporreicas (aguas subterráneas) y cuya concentración de nutrientes esta en concentraciones no detectables limitando el desarrollo de especies sensibles a contaminación ${ }^{(14,15)}$. En caso del LG su elevado índice de diversidad (mayor de 1 bits. cél-1) está constituido por especies tolerantes a concentraciones elevadas de dureza, conductividad, DQO, $\mathrm{DBO}_{5}$ y coliformes totales ${ }^{(22,23)}$.

La información obtenida es útil debido a que resume el comportamiento de la calidad del agua basándose en el ensamblaje de las comunidades de diatomeas y su estrecha relación con sus parámetros ambientales y clasificando a la cuenca en ambientes sanos (desde $\mathrm{CH}$ hasta TA), medianamente contaminados (CA y SA) y fuertemente contaminados (desde $Y L$ hasta $L G$ ), a diferencia de la información que proporciona la nube de datos fisicoquímicos que por sí sola es muy dispersa, y muchas veces no se puede interpretar de manera global ${ }^{(23)}$. En nuestro estudio la principal limitación fue la escasa información sobre la identificación y autoecología de las diatomeas continentales en los ríos de las zonas costeras y altoandinas del Perú.

En conclusión, encontramos que existe un incremento en el grado de contaminación en el rio Locumba que se hace manifiesto con el descenso altitudinal a lo largo de su cuenca, ello fue evidente gracias a los parámetros fisicoquímicos y microbiológicos tradicionalmente empleados para este tipo de determinación; de igual forma, dicha contaminación se manifestó no solo con la disminución de la biodiversidad de las diatomeas sino además con alteraciones en el ensamblaje de estas en función a las características de su entorno, pues se encontró especies de diatomeas que son características de ambientes contaminados. Por ello, consideramos que el uso de bioindicadores, como el índice de diversidad de Shanon-Wienner para diatomeas podría ser empleado para la evaluación de la calidad de agua de los ríos en el Perú. Se recomienda estudiar ecosistemas similares y desarrollar una base de datos referencial para cada cuenca, como una herramienta de gestión de los recursos hídricos.

Contribución de autoría: JACA participó en la concepción y diseño del artículo, análisis e interpretación de datos y asesoría estadística. MAC participó en la realización de análisis químicos complementarios. SDV participó en la asesoría administrativa. Todos los autores participaron en la revisión crítica del artículo y aprobación de su versión final.

Fuentes de financiamiento: Oficina General de Investigación y Transferencia Tecnológica (Referencia: RT694-2005 OPD/INS).

Conflictos de interés: los autores declaran no tener conflictos de interés.

\section{REFERENCIAS BIBLIOGRÁFICAS}

1. Rivera-Rondón CA, Díaz-Quirós, C. Grandes taxones de fitobentos y su relación con la hidrología, física y química de pequeños ríos andinos. Universitas Scientiarum. 2004;9(1):75-86.

2. Directiva $2000 / 60 / \mathrm{CE}$ del Parlamento Europeo y del Consejo, de 23 de octubre de 2000, por la que se establece un marco comunitario de actuación en el ámbito de la política de aguas [Internet]. EUR-Lex; c2013 [citado el 15 de enero de 2011]. Disponible en: http://eurlex.europa.eu/LexUriServ/LexUriServ. do?uri=CELEX:32000L0060:ES:NOT

3. Della Bell V, Puccinelli C, Marcheggiani $S$, Mancini L. Benthic diatom communities and their relationship to water che- mistry in wetlands of central Italy. Ann Limnol-Int J Lim. 2007;43(2):89-99.

4. Cox E. What in the basis for using diatoms as monitors of river quality? En: Whitton BA, Rott E, Friedrich G, editors. Use of algae for monitoring rivers. Innsbruck: Institut für Botanik, Universität Innsbruck; 1991. p. 33-40.

5. McCormick PV, Cairns J Jr. Algae as indicators of environmental change. J Appl Phycol. 1994;6:509-526.

6. Margalef R. Los Organismos Indicadores en la Limnologia. Tipografía artística. Madrid: Instituto Forestal de Investigaciones y Experiencias; 1955.

7. Kelly MG, Cazaubon A, Coring E, Dell'Uomo A, Ector, L, Goldsmith B, et al.
Recommendations for the routine sampling of diatoms for water quality assessments in Europe.J Appl Phycol. 1998;10(2):215-24.

8. European Committee for Standardization. Water quality - Guidance standard for the routine sampling and pretreatment of benthic diatoms from rivers. London: British Standard; 2002.

9. Krammer K, Lange-Bertalot H. Bacillariophyceae: Naviculaceae. In: Ettl H, Gerloff J, Heynig H, Mollenhauer D (eds). Süßwasserflora von Mitteleuropa. Stuttgart: G. Fischer; 1986. p. 876.

10. Krammer K, Lange-Bertalot H. Bacillariophyceae: Bacillariaceae, Epithemiaceae, Surirellaceae. Ettl H, Gerloff J, Heynig H, Mollenhauer D (eds). 
Süßwasserflora von Mitteleuropa. Stuttgart: G. Fischer; 1988. p. 595.

11. Krammer K, Lange-Bertalot H. Bacillariophyceae: Centrales, Fragilariaceae, Eunotiaceae. In: Ettl H, Gerloff J, Heynig H, Mollenhauer D (eds). Süßwasserflora von Mitteleuropa. Stuttgart: G. Fischer; 1991. p. 576.

12. Krammer K, Lange-Bertalot H. Bacillariophyceae: Achnanthaceae, Kritische Ergazungen zu Navicula (Lineolatae) und Gomphonema. In: Ettl H, Gerloff J, Heynig H, Mollenhauer D (eds). Süßwasserflora von Mitteleuropa. Stuttgart: G. Fischer; 1991b. 437 pp.

13. Rumrich U, Lange-Bertalot $\mathrm{H}$, Rumrich M. Diatomeen der Anden: von Venezuela bis Patagonien/Feuerland: und zwei weitere Beiträge. Iconographia Diatomologica 9. Königstein, Germany: A.R.G. Gantner; 2000.

14. Rakowska B. Indicatory values in ecological description of diatoms from Polish lowlands. Ecohydrology and Hydrobiology. 2001;1(4):481-502.
15. Gomez N. Use of epipelic diatoms for evaluation of water quality in the Matanza-Riachuelo (Argentina), a pampean plain river. Water research. 1998;32(7):2029-34.

16. Allan D. Stream Ecology. Structure and function of running waters. London: Chapman \& Hall; 1995.

17. Castro de Esparza ML. Arsénico en el agua de bebida de América Latina y su efecto en la salud pública. Hojas de Divulgación Técnica. Lima: OPS-Perú; 2004.

18. Nielsen DL, Brock MA, Rees GN, Baldwin DS. Effects of increasing salinity on freshwater ecosystem in Australia. Aust J Bot. 2003;51:655-65.

19. Hidalgo MV, Meoni GS, Barrionuevo MA, Navarro G, Paz R. Variabilidad de la relación $\mathrm{DBO}_{5} / \mathrm{DQO}$ en ríos de Tucuman, Argentina. $13^{\circ}$ Congreso Argentino de Saneamiento y Medio Ambiente. Buenos Aires, Argentina, 09 al 11 de setiembre del 2003.

20. Lobo E, Kobayasi H. Shannon's diversity index applied to some freshwater diatom assemblages in the Sa- kawa River System (Kanagawa Pref., Japan) and its use as an indicator of water quality. Jpn J Phycol (Sorui). 1990;38:229-43.

21. Xiangdong Y, Sumin W, Weilan X, Wanchun L. Application of CCA for study on modern lake diatoms and environment in the Tibetan Plateau. Science in China. 2001;44 Suppl 1:343-50.

22. Lange-Bertalot H. Pollution tolerance of diatoms as a criterion for water quality estimation. Nova Hedwigia Beiheft. 1979;64:285-304.

23. Gómez N, Licursi M. The Pampean Diatom Index (IDP) for assessment of rivers and streams in Argentina. Aquat Ecol. 2001;35(2):173-81.

Correspondencia: José Alberto Calizaya Anco Dirección: Hábitat Los Rosales R-05, Tacna, Perú. Teléfono: (052) 317072 / 952842438

Correo electrónico:josecalizaya2011@gmail.com

\section{Consulte las ediciones anteriores de la Revista Peruana de Medicina Experimental y Salud Pública en}

\section{wWw.scielosp.org}

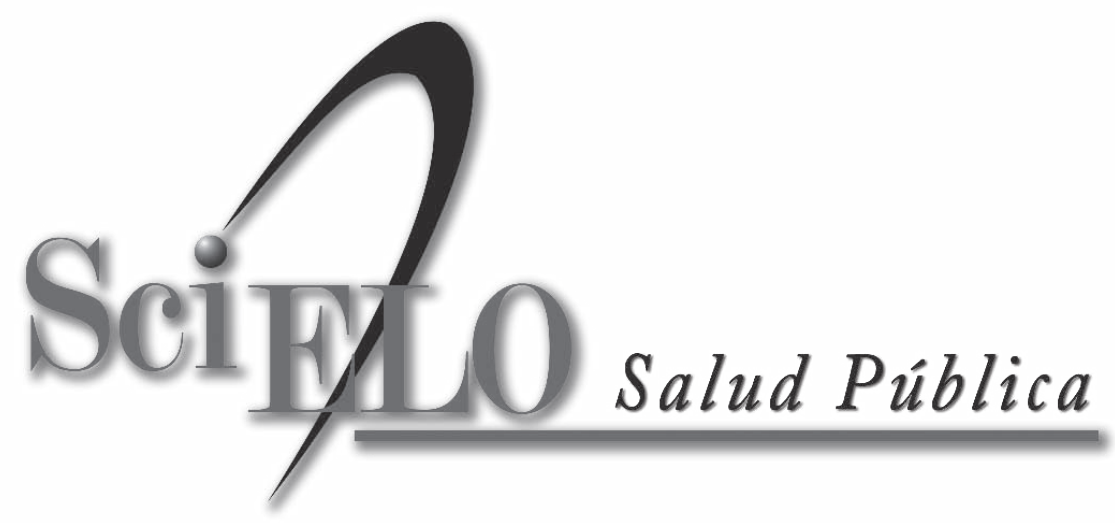

